Supporting Information

\title{
Electric-Field-Induced Chirality in Columnar Liquid Crystals
}

Alberto Concellón, Ru-Qiang Lu, Kosuke Yoshinaga, Hsiu-Fu Hsu, and Timothy M. Swager*

Department of Chemistry, Massachusetts Institute of Technology, 77 Massachusetts Avenue, Cambridge, Massachusetts 02139, United States

*Author for correspondence: tswager@mit.edu 


\section{CONTENTS}

1. Materials and Characterization Techniques

1.1. Materials

1.2. Characterization Techniques

1.3. Liquid Crystal Switching Experiments

1.4. Theoretical Calculations

2. Synthesis and Characterization

3. Supplementary Figures 


\section{MATERIALS AND METHODS}

\subsection{Materials}

3,4-Bis(octyloxy)phenylboronic acid, ${ }^{1}$ and (S)-(3-fluoro-4-(octan-2-yloxy)phenyl)boronic $\mathrm{acid}^{2}$ were prepared following previously reported procedures. Commercial reagents were used as received without further purification: 1,4-dibromo-2,5-diiodobenzene, Tetrakis(triphenylphosphine)palladium(0) (Sigma-Aldrich); dichloromethane, tetrahydrofuran, methanol, ethanol, toluene, acetone, ethyl acetate (VWR). Anhydrous toluene was purchased from Sigma-Aldrich and dried using an INERT PureSolv MD5 solvent purification system. Deuterated solvents were purchased from Cambridge Isotope Laboratories and used as received.

\subsection{Characterization Techniques}

ATR-FTIR spectra were obtained on a Thermo Scientific Nicolet 6700 FTIR spectrophotometer with a Ge crystal for ATR. Solution NMR experiments were carried out on Bruker Avance spectrometers operating at $400 \mathrm{MHz}$ for ${ }^{1} \mathrm{H}$ and $100 \mathrm{MHz}$ for ${ }^{13} \mathrm{C}$, using standard pulse sequences. Chemical shifts are given in ppm relative to TMS and the residual solvent peak was used as internal reference. High-resolution mass spectra (HRMS) were performed on a JEOL AccuTOF-DART mass spectrometer.

Thermogravimetric analysis (TGA) was performed using a Q50 from TA instruments at heating rate of $10^{\circ} \mathrm{C} / \mathrm{min}$ under a nitrogen atmosphere. Liquid crystal properties were investigated by polarized-light optical microscopy (POM) using a Leica DMRXP polarized-light microscope fitted with a Linkam TMS 94 hot stage. Thermal transitions were determined by differential scanning calorimetry (DSC) using a Discovery DSC from TA instruments with powdered samples (2-5 mg) sealed in aluminum pans. First order transition temperatures were read at the maximum of the corresponding peak. X-ray diffraction (XRD) was performed with a SAXSLAB instrument equipped with a Rigaku 002 microfocus X-ray source $\left(\mathrm{CuK}_{\mathrm{a} 1}=1.5409 \AA\right)$ and a Dectris Pilatus $300 \mathrm{~K}$ detector. The beam center and the $q$ range were calibrated using the diffraction peaks of silver behenate. Powdered samples were placed in Lindemann glass capillaries $(1 \mathrm{~mm}$ diameter).

UV-Vis absorption spectra were recorded on a Cary 60 spectrophotometer. Fluorescence measurements were performed using a Horiba Quanta- $\phi$ fluorescence spectrophotometer.

Circular dichroism (CD) spectra were recorded at room temperature on a JASCO J-1500 Circular Dichroism Spectrophotometer. CD spectra recorded at different rotation angles

\footnotetext{
${ }^{1}$ Zhang, Q.; Peng, H.; Zhang, G.; Lu, Q.; Chang, J.; Dong, Y.; Shi, X.; Wei, J. J. Am. Chem. Soc. 2014, 136 (13), 5057-5064

${ }^{2}$ Chen, Y.-H.; Liu, S.-F.; Li, H.-A.; Fuh, S.-M.; Lin, H.-C.; Chen, H.-M.; Yang, P.-J.; Chien, S.-C. US Pat. Appl. Publ. $20120289731,2012$.
} 
around the light beam showed the same trace and were averaged in order to compensate linear dichroism artifacts. ${ }^{3}$

\subsection{Liquid Crystal Switching Experiments}

Liquid crystal test cells were prepared by overlapping two ITO-coated quartz substrates controlling the spacing between the two substrates with $5 \mu \mathrm{m}$ glass spacers. The quartz substrates with ITO electrodes (8-12 ohms, $300 \mathrm{~nm}$ thick ITO) were purchased from UQG Optics. The thickness of the cells, measured by interferometry, ranged from 5 to 8 $\mu \mathrm{m}$. To prepare the samples, discotic mesogens were heated to isotropic and loaded into a warm cell via capillary action. Because of the high viscosity of the materials, it was necessary to wait around 2 hours to observe a complete cell filling. Then, samples were cooled to room temperature at $1{ }^{\circ} \mathrm{C} / \mathrm{min}$. For the E-field-induced chirality experiments, direct current (DC) fields with various voltages were directly applied to the ITO liquid crystal cells. The electric fields were generated with a DDS dual-channel function signal source generator (FeelTech) and amplified with a 6827A bipolar power supply/amplifier (Hewlett Packard).

\subsection{Theoretical Calculations}

Geometric optimizations of four conformers of 3 in the gas phase were carried out at the B3LYP-D3/6-31g(d) level on Gaussian 9, Revision D.01. ${ }^{4}$ Harmonic vibrational frequency calculations were performed for all of the stationary points to confirm them as local minima. The side $-\mathrm{OC}_{8} \mathrm{H}_{17}$ chains were replaced by shorter $-\mathrm{OC}_{4} \mathrm{H}_{9}$ chains to simplify the calculations. PM7 semiempirical calculations of octamer of 3-1 were carried out on MOPAC2016. ${ }^{5}$

\footnotetext{
${ }^{3}$ Gottarelli, G.; Lena, S.; Masiero, S.; Pieraccini, S.; Spada, G. P. Chirality 2008, 20 (3-4), 471-485.

${ }^{4}$ Gaussian 09, Revision D.01, M. J. Frisch, G. W. Trucks, H. B. Schlegel, G. E. Scuseria, M. A. Robb, J. R. Cheeseman, G. Scalmani, V. Barone, B. Mennucci, G. A. Petersson, H. Nakatsuji, M. Caricato, X. Li, H. P. Hratchian, A. F. Izmaylov, J. Bloino, G. Zheng, J. L. Sonnenberg, M. Hada, M. Ehara, K. Toyota, R. Fukuda, J. Hasegawa, M. Ishida, T. Nakajima, Y. Honda, O. Kitao, H. Nakai, T. Vreven, J. A. Montgomery, Jr., J. E. Peralta, F. Ogliaro, M. Bearpark, J. J. Heyd, E. Brothers, K. N. Kudin, V. N. Staroverov, T. Keith, R. Kobayashi, J. Normand, K. Raghavachari, A. Rendell, J. C. Burant, S. S. Iyengar, J. Tomasi, M. Cossi, N. Rega, J. M. Millam, M. Klene, J. E. Knox, J. B. Cross, V. Bakken, C. Adamo, J. Jaramillo, R. Gomperts, R. E. Stratmann, O. Yazyev, A. J. Austin, R. Cammi, C. Pomelli, J. W. Ochterski, R. L. Martin, K. Morokuma, V. G. Zakrzewski, G. A. Voth, P. Salvador, J. J. Dannenberg, S. Dapprich, A. D. Daniels, O. Farkas, J. B. Foresman, J. V. Ortiz, J. Cioslowski, and D. J. Fox, Gaussian, Inc., Wallingford CT, 2013.

${ }^{5}$ MOPAC2016 and J. J. P. Stewart, Stewart Computational Chemistry, Colorado Springs, CO, USA, HTTP://OpenMOPAC.net.
} 


\section{SYNTHESIS AND CHARACTERIZATION}
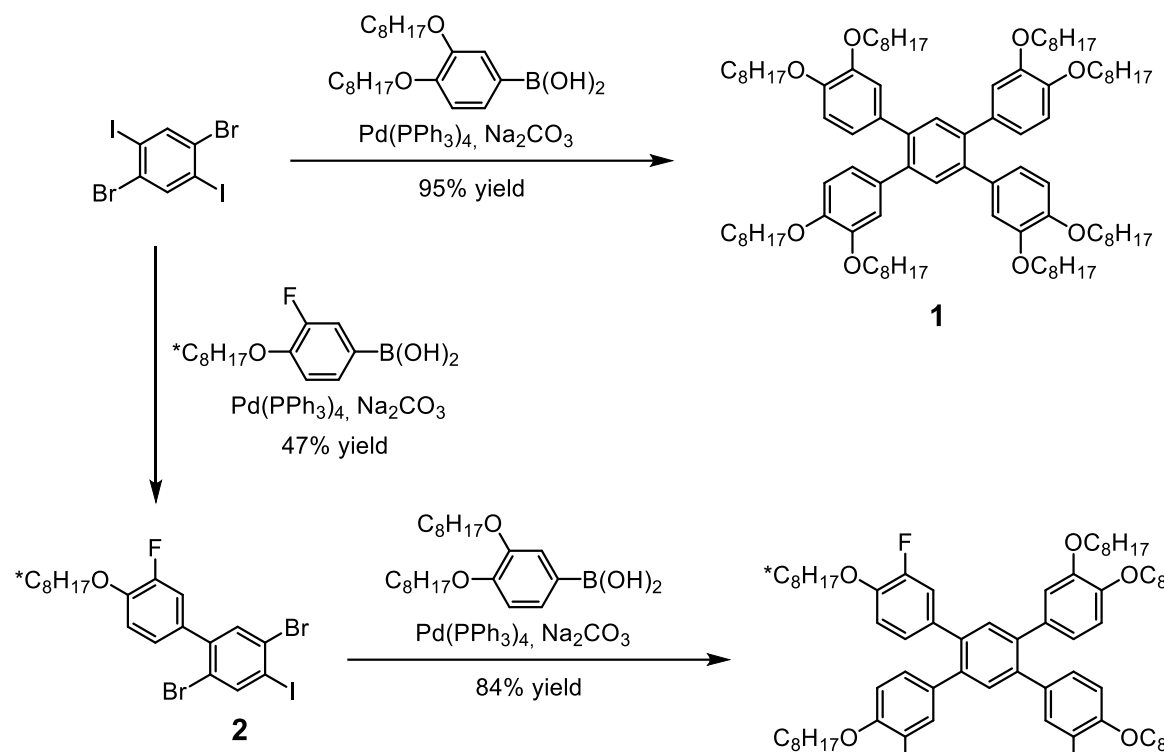

1

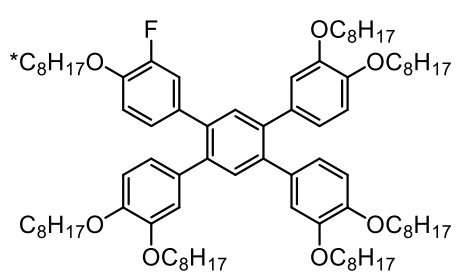

3

Discotic mesogen 1. 2,4-Dibromo-1,5-diiodobenzene (43 mg, $0.09 \mathrm{mmol}$ ), 3,4bis(octyloxy)phenylboronic acid $(150 \mathrm{mg}, 0.40 \mathrm{mmol}), \quad$ palladium(0) (tetrakistriphenylphosphine) $(5.1 \mathrm{mg}, 0.0044 \mathrm{mmol})$, and sodium carbonate ( $94 \mathrm{mg}$, $0.88 \mathrm{mmol}$ ) were suspended in a mixture of dry toluene $(10 \mathrm{~mL})$ in a Schleck flask. The mixture was made to react under argon atmosphere at $80{ }^{\circ} \mathrm{C}$ for $48 \mathrm{~h}$. The reaction was allowed to cool down to room temperature and poured into water and extracted three times with diethyl ether. The combined organic phases were washed with water and brine and dried over anhydrous magnesium sulfate. The solution was filtered and the solvent was removed under reduced pressure. The resulting product was purified with column chromatography on silica gel using DCM/hexanes (1:4) as eluent in a Biotage Isolera. Yield: $95 \%$. IR $\left(\mathrm{v}, \mathrm{cm}^{-1}\right): 2920,1602,1578,1521,1467$, 1249, 1137, 1016, 806. ${ }^{1} \mathrm{H}$ NMR ( $\left.\mathrm{CDCl}_{3}, 400 \mathrm{MHz}, \delta, \mathrm{ppm}\right): 7.50$ (s, 2H), 6.83-6.74 $(\mathrm{m}, 8 \mathrm{H}), 6.71-6.66(\mathrm{~m}, 4 \mathrm{H}), 3.95(\mathrm{t}, J=6.8 \mathrm{~Hz}, 8 \mathrm{H}), 3.70(\mathrm{t}, J=6.8 \mathrm{~Hz}, 8 \mathrm{H}), 1.80(\mathrm{q}$, $\mathrm{J}=7.1 \mathrm{~Hz}, 8 \mathrm{H}), 1.66(\mathrm{q}, \mathrm{J}=7.2 \mathrm{~Hz}, 8 \mathrm{H}), 1.51-1.20(\mathrm{~m}, 80 \mathrm{H}), 0.96-0.83(\mathrm{~m}, 24 \mathrm{H}) .{ }^{13} \mathrm{C}$ NMR $\left(\mathrm{CDCl}_{3}, 100 \mathrm{MHz}, \delta\right.$, ppm): 148.61, 148.09, 139.19, 134.10, 132.47, 122.11, 116.00, 113.47, 69.40, 69.22, 32.00, 29.59, 29.52, 29.49, 29.45, 29.27, 26.22, 26.17, 22.85, 22.83, 14.25. HRMS (MALDI): $\mathrm{m} / \mathrm{z}$ calcd. for $\mathrm{C}_{94} \mathrm{H}_{150} \mathrm{O}_{8}[\mathrm{M}]^{+}, 1407.1331$; found, 1407.1344 .

Precursor 2. 2,4-Dibromo-1,5-diiodobenzene (363.8 mg, $0.75 \mathrm{mmol}),(S)$ - or $(R)$ (3-fluoro-4-(octan-2-yloxy)phenyl)boronic acid (200 mg, $0.75 \mathrm{mmol}$ ), palladium(0) (tetrakistriphenylphosphine) $(43.1 \mathrm{mg}, 0.04 \mathrm{mmol}$ ), and sodium carbonate (200 mg, $1.86 \mathrm{mmol})$ were suspended in a mixture of dry toluene $(10 \mathrm{~mL})$ in a Schleck flask. The mixture was made to react under argon atmosphere at $80^{\circ} \mathrm{C}$ for $48 \mathrm{~h}$. The reaction was allowed to cool down to room temperature and poured into water and extracted three times with diethyl ether. The combined organic phases were washed with water and brine and dried over anhydrous magnesium sulfate. The solution was filtered and the solvent was removed under reduced pressure. The resulting product 
was purified with column chromatography on silica gel using DCM/hexanes (1:5) as eluent in a Biotage Isolera. Yield: $40-47 \%$. IR $\left(\mathrm{v}, \mathrm{cm}^{-1}\right): 2954,1618,1570,1519,1468$, 1268, 1129, 1080, 1026. ${ }^{1} \mathrm{H}$ NMR $\left(\mathrm{CDCl}_{3}, 400 \mathrm{MHz}, \delta, \mathrm{ppm}\right): 8.12$ (s, 1H), 7.55 (s, $1 \mathrm{H}), 7.18-6.80(\mathrm{~m}, 3 \mathrm{H}), 4.47-4.37(\mathrm{~m}, 1 \mathrm{H}), 1.91-1.16(\mathrm{~m}, 13 \mathrm{H}), 0.89(\mathrm{t}, J=6.8 \mathrm{~Hz}$, $3 \mathrm{H}) .{ }^{13} \mathrm{C} \mathrm{NMR}\left(\mathrm{CDCl}_{3}, 100 \mathrm{MHz}, \delta, \mathrm{ppm}\right): 152.9(\mathrm{~d}, J=246.5 \mathrm{~Hz}), 146.49$ (d, J=10.5 $\mathrm{Hz}), 143.50,134.41,131.82,128.96,125.17$ (d, J=3.6 Hz), 121.80, 117.48 (d, J= $10.0 \mathrm{~Hz}), 116.63,100.30,76.37,26.64,36.61,31.93,29.39,25.61,22.74,19.96$, 14.23 .

Discotic mesogen 3. Precursor (R)-2 or (S)-2 (50 mg, $0.11 \mathrm{mmol}$ ), 3,4bis(octyloxy)phenylboronic acid (146 $\mathrm{mg}, 0.38 \mathrm{mmol}), \quad$ palladium(0) (tetrakistriphenylphosphine) $(6.4 \mathrm{mg}, 0.0055 \mathrm{mmol})$, and sodium carbonate (93.2 $\mathrm{mg}$, $0.88 \mathrm{mmol})$ were suspended in a mixture of dry toluene $(10 \mathrm{~mL})$ in a Schleck flask. The mixture was made to react under argon atmosphere at $80{ }^{\circ} \mathrm{C}$ for $48 \mathrm{~h}$. The reaction was allowed to cool down to room temperature and poured into water and extracted three times with diethyl ether. The combined organic phases were washed with water and brine and dried over anhydrous magnesium sulfate. The solution was filtered and the solvent was removed under reduced pressure. The resulting product was purified with column chromatography on silica gel using DCM/hexanes (1:1) as eluent in a Biotage Isolera. Yield: $77-84 \%$. IR $\left(\mathrm{v}, \mathrm{cm}^{-1}\right): 2920,1602,1576,1512,1467$, 1249, 1138, 1016, 808. ${ }^{1} \mathrm{H}$ NMR $\left(\mathrm{CDCl}_{3}, 400 \mathrm{MHz}, \delta, \mathrm{ppm}\right): 7.52(\mathrm{~s}, 1 \mathrm{H}), 7.47$ (s, $1 \mathrm{H}), 7.01-6.63(\mathrm{~m}, 12 \mathrm{H}), 4.36-4.26(\mathrm{~m}, 1 \mathrm{H}), 4.02-3.90(\mathrm{~m}, 6 \mathrm{H}), 3.79-3.62(\mathrm{~m}, 6 \mathrm{H})$, 1.88-1.74 (m, 8H), 1.73-1.20 (m, 80H), 0.98-0.83 (m, 21H). ${ }^{13} \mathrm{C} \mathrm{NMR}\left(\mathrm{CDCl}_{3}, 100\right.$ $\mathrm{MHz}, \delta, \mathrm{ppm}): 154.05$ (d, J=243 Hz), 148.62, 148.15, 144.92 (d, J=10.4 Hz), 139.57, 139.28, 133.95, 133.61, 132.57, 125.68, 122.10, 117.97 (d, J= $7.9 \mathrm{~Hz}), 117.02$, $115.99,113.51,76.37,69.39,69.23,36.67,31.99,31.97,31.95,29.58,29.56,29.52$, $29.49,29.44,29.27,26.21,26.16,25.63,22.83,22.75,19.93,14.25,14.22$. HRMS (MALDI): $\mathrm{m} / \mathrm{z}$ calcd. for $\mathrm{C}_{86} \mathrm{H}_{133} \mathrm{FO}_{7}\left[\mathrm{M}^{+}, 1297.0035\right.$; found, 1297.0019 . 


\section{SUPPLEMENTARY FIGURES}
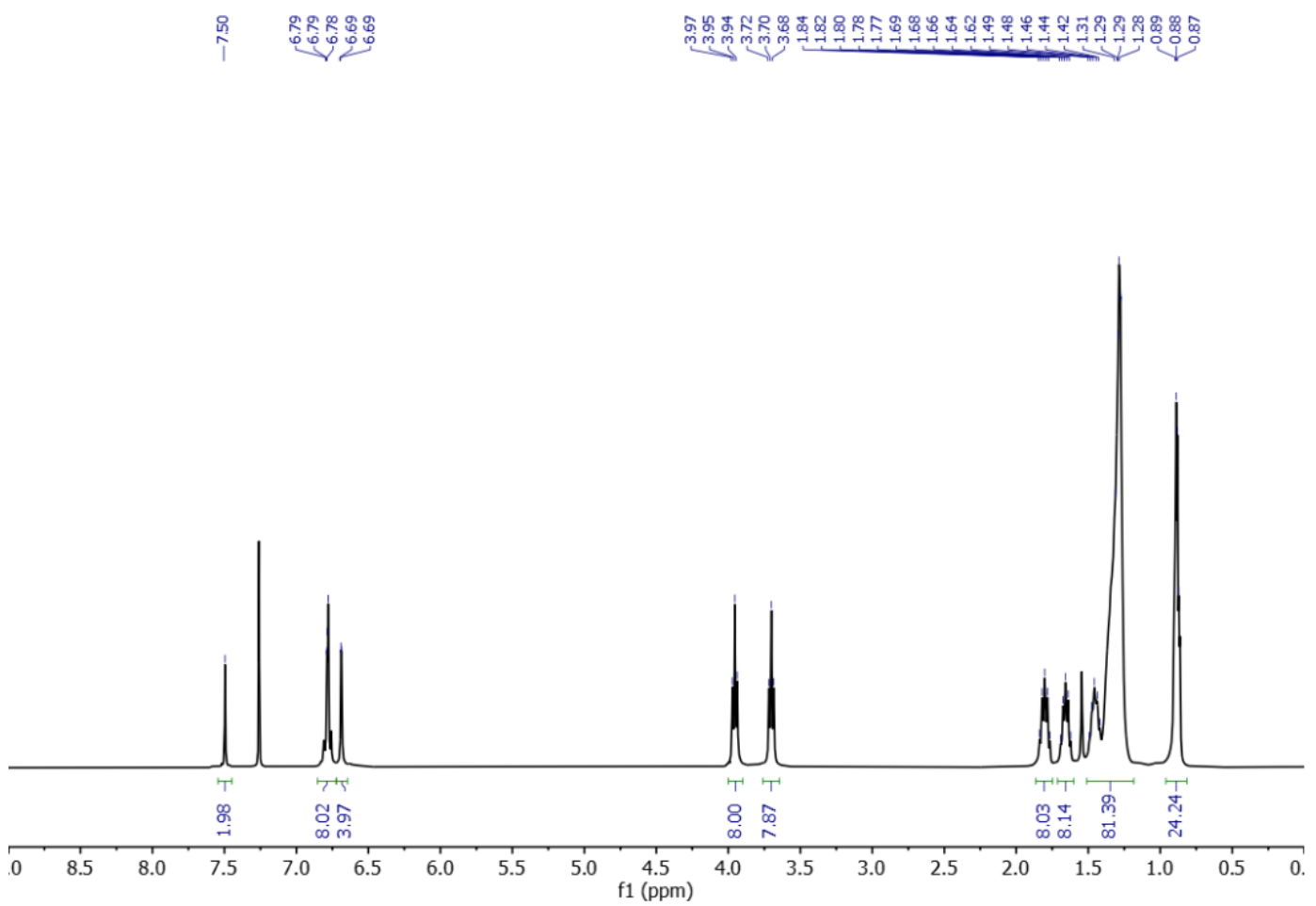

Figure S1. ${ }^{1} \mathrm{H}$ NMR spectrum $\left(400 \mathrm{MHz}, 298 \mathrm{~K}, \mathrm{CDCl}_{3}\right)$ of 1.

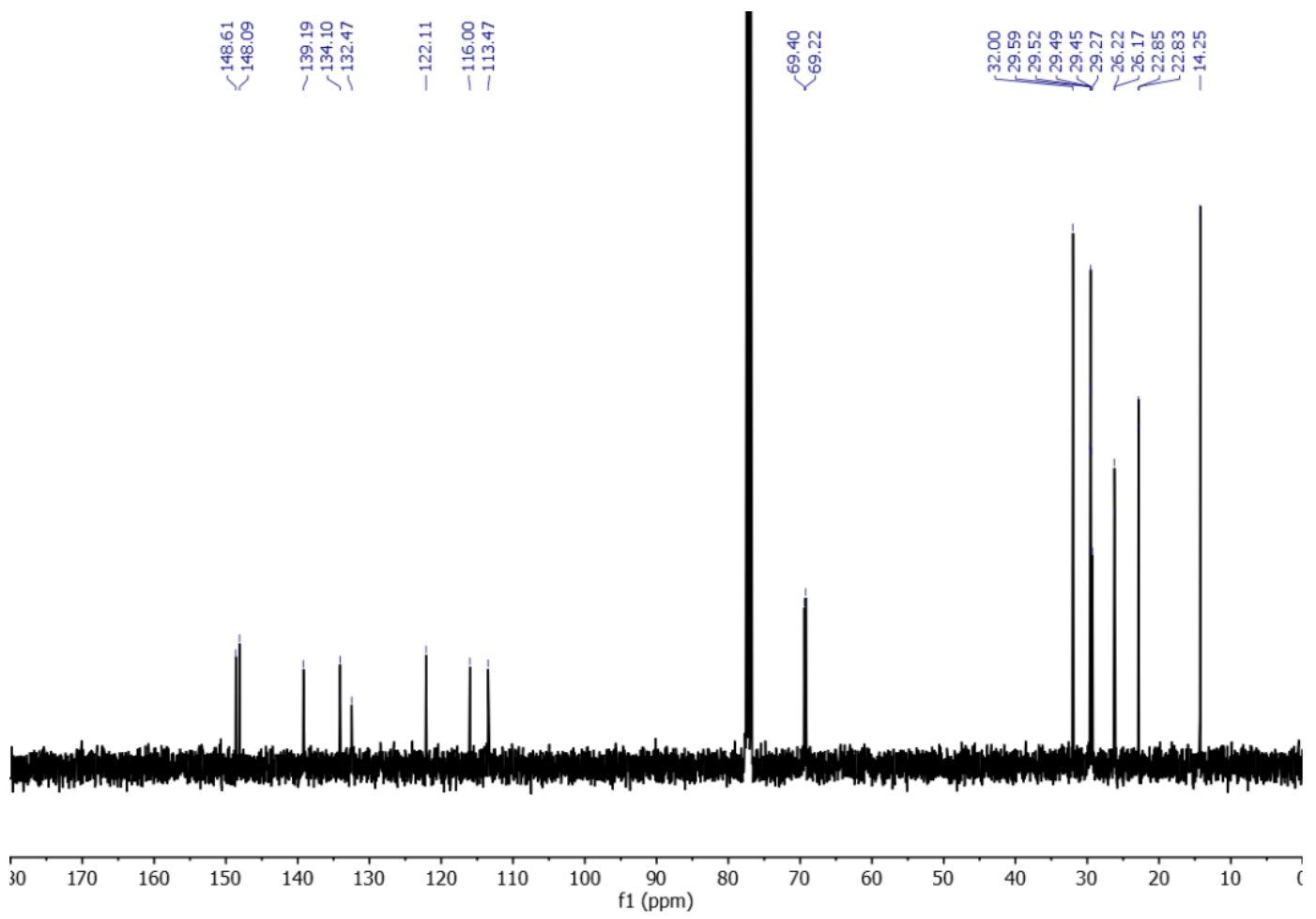

Figure S2. ${ }^{13} \mathrm{C}$ NMR spectrum $\left(100 \mathrm{MHz}, 298 \mathrm{~K}, \mathrm{CDCl}_{3}\right)$ of 1. 


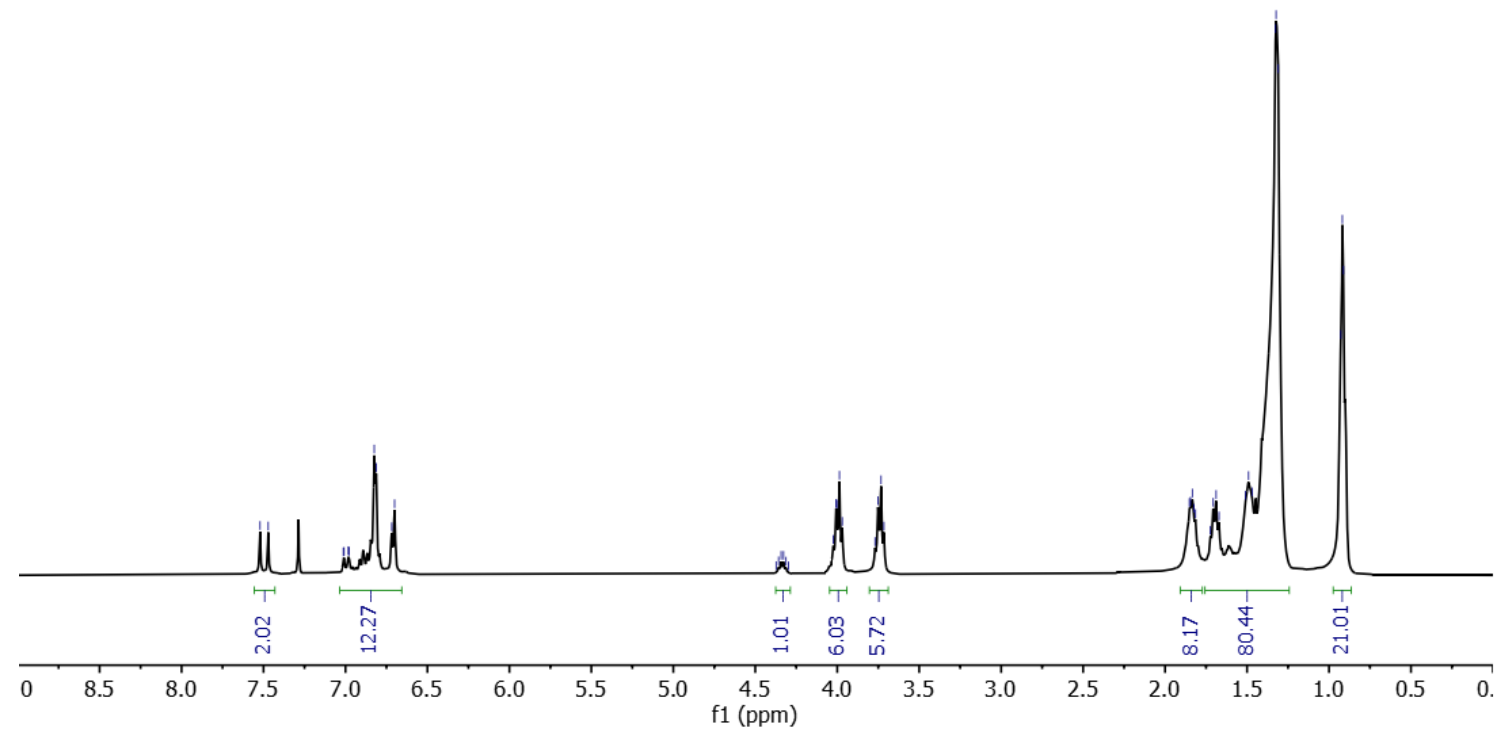

Figure S3. ${ }^{1} \mathrm{H}$ NMR spectrum (400 MHz, $298 \mathrm{~K}, \mathrm{CDCl}_{3}$ ) of (S)-3.

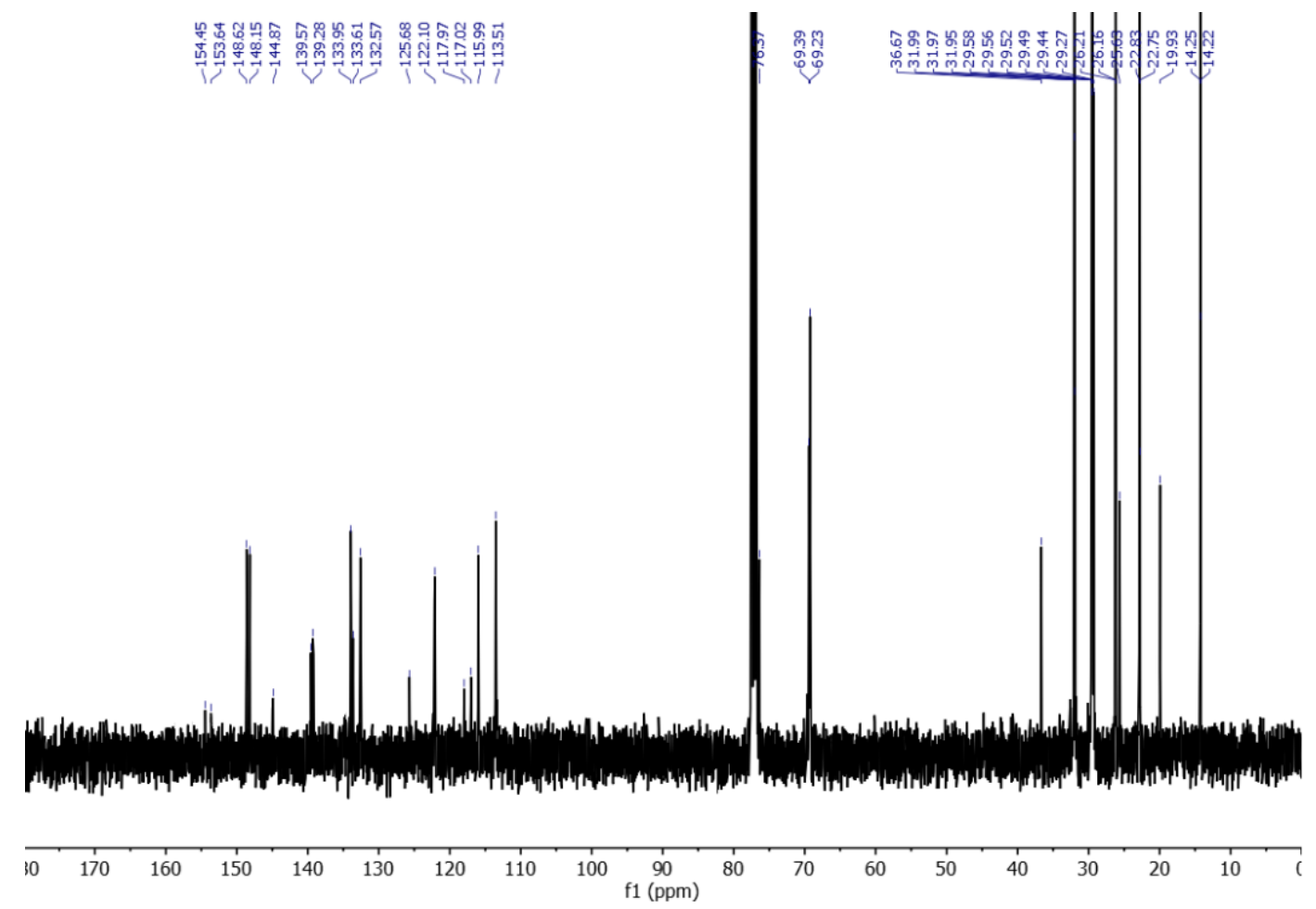

Figure S4. ${ }^{13} \mathrm{C}$ NMR spectrum (100 MHz, 298K, CDCl 3 ) of (S)-3. 


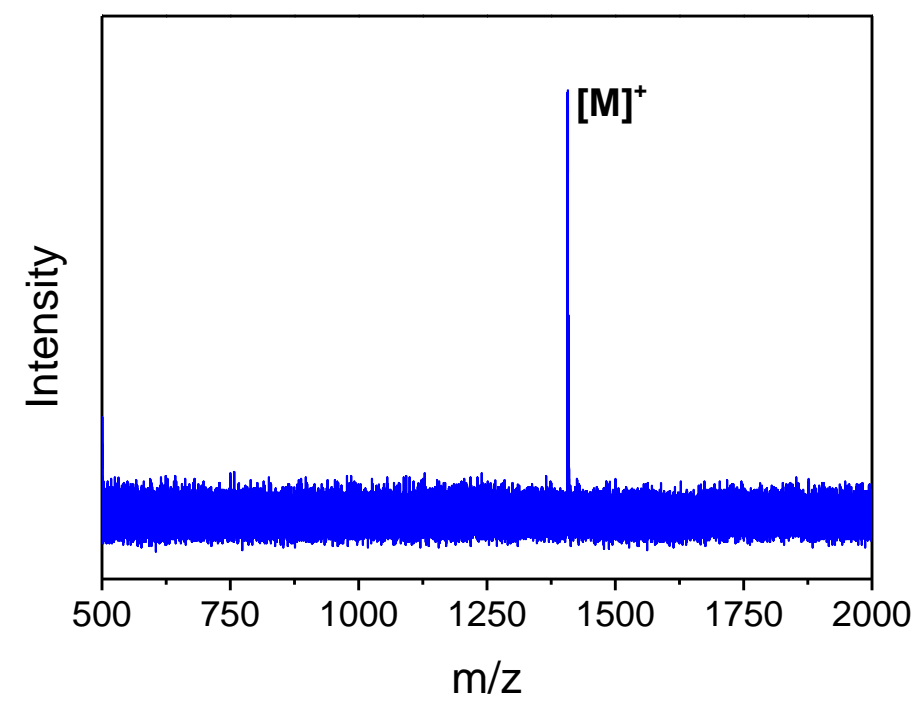

Figure S5. MALDI-TOF mass spectrum (MALDI+, DIT) of 1.

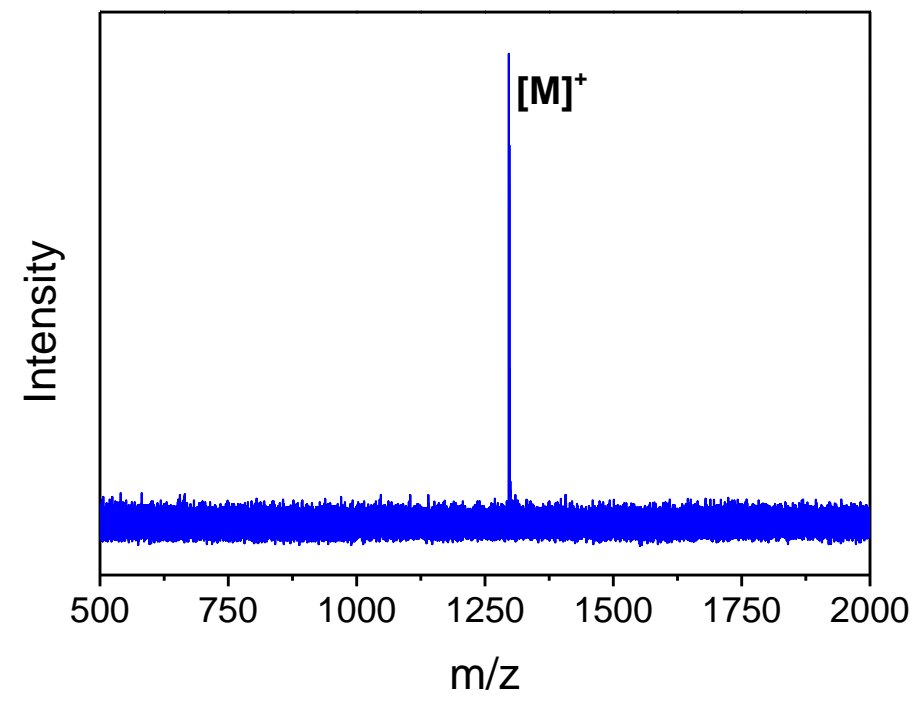

Figure S6. MALDI-TOF mass spectrum (MALDI+, DIT) of (S)-3. 


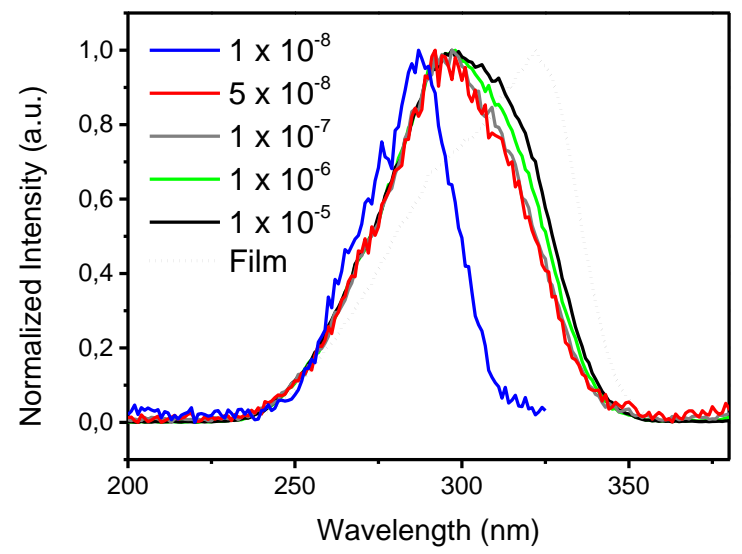

Figure S7. Normalized concentration-dependent changes in excitation spectra of $(S)$-3 in chloroform solutions and thin film.

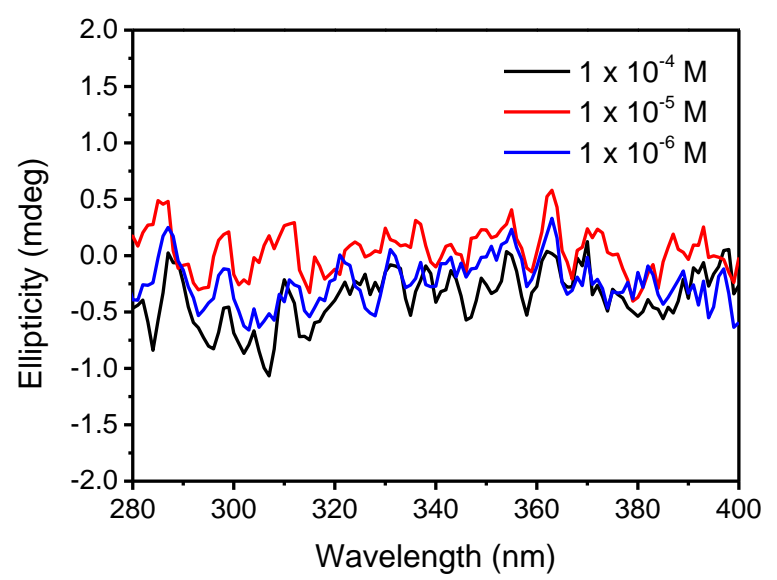

Figure S8. CD spectra in chloroform solution of (S)-3.

(a)

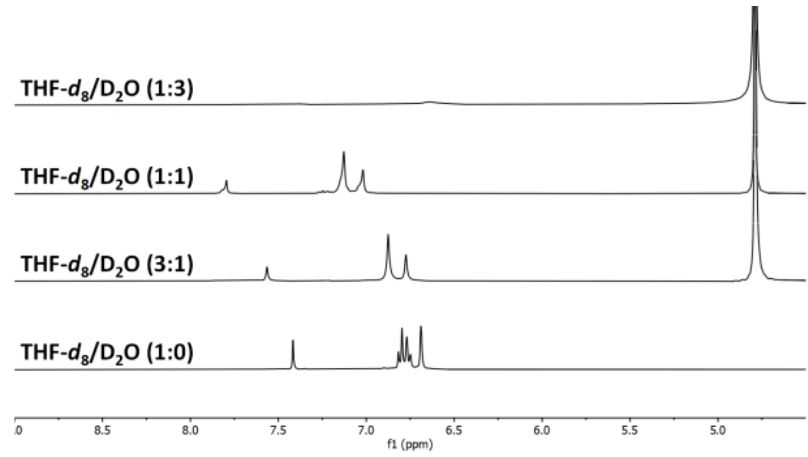

(b)

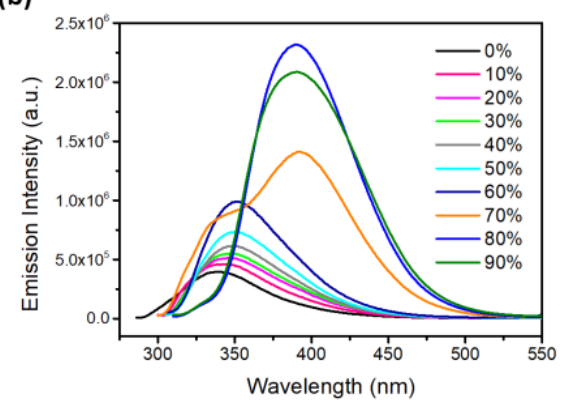

Figure S9. (a) ${ }^{1} \mathrm{H}$ NMR spectra recorded for 1 dissolved in THF- $d_{8} / \mathrm{D}_{2} \mathrm{O}$ mixtures, wherein the upfield shifting of the aromatic signals suggest self-assembly via $\pi-\pi$ interactions. (b) Emission spectra of (S)-3 in tetrahydrofuran/water mixtures. 


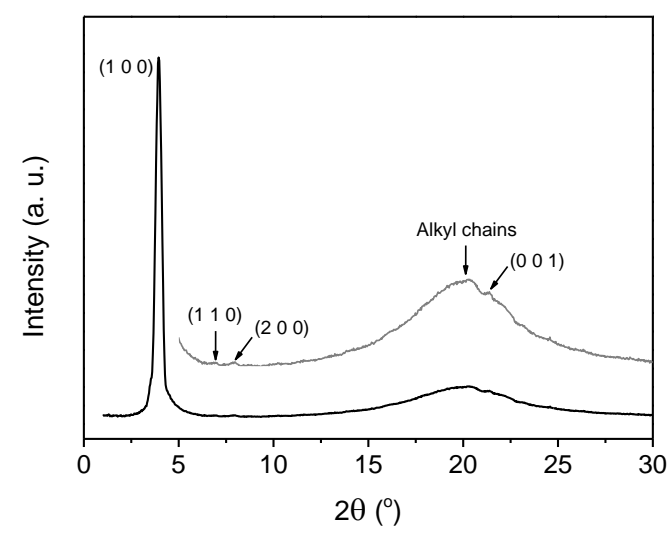

Figure S10. 1D XRD profile of 1.
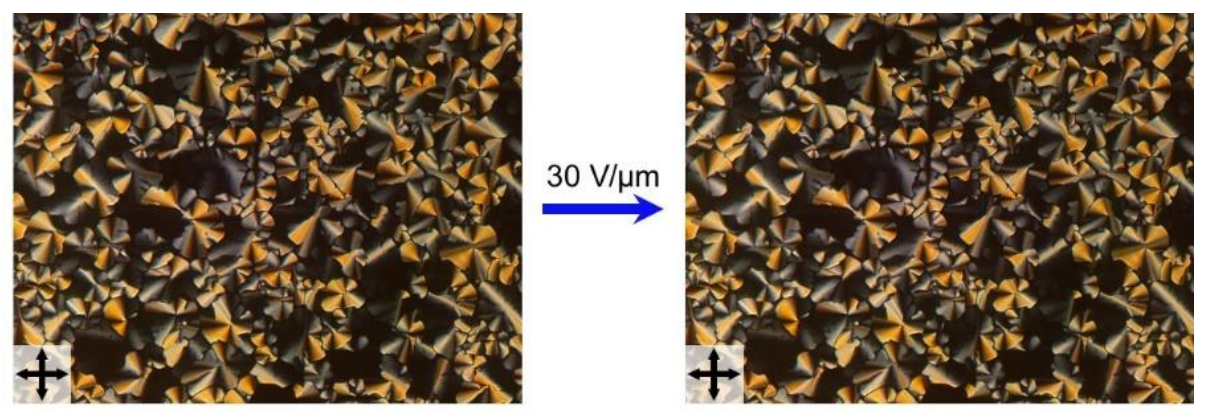

Figure S11. POM images obtained from LC cells of discotic mesogen 1 in the hexagonal columnar phase under: (a) Zero field, and (b) a DC electric field (30 V/ $\mu \mathrm{m})$.

(a)

\begin{tabular}{|c|c|c|c|}
\hline $\begin{array}{c}\text { Mole fraction } \\
\text { of (S)-3 in } 1\end{array}$ & $\mathrm{~T}_{2 \%}\left({ }^{\circ} \mathrm{C}\right)$ & LC properties ${ }^{[a]}$ & XRD parameters ${ }^{[c]}$ \\
\hline 0 & 263 & $\mathrm{Col}_{\mathrm{h}} 79 \mathrm{I}$ & $\mathrm{a}=25.9 \AA ; \mathrm{c}=4.0 \AA$ \\
\hline 0,25 & 231 & $\mathrm{Col}_{\mathrm{h}} 68 \mathrm{I}$ & $\mathrm{a}=25.8 \AA ; \mathrm{c}=3.9 \AA$ \\
\hline 0,50 & 220 & $\mathrm{Col}_{\mathrm{h}} 60 \mathrm{I}$ & $a=25.9 \AA ; c=4.0 \AA$ \\
\hline 0,75 & 246 & $\mathrm{Col}_{\mathrm{h}} 55 \mathrm{I}$ & $\mathrm{a}=25.9 \AA ; \mathrm{c}=3.8 \AA$ \\
\hline 1 & 245 & $\mathrm{Col}_{\mathrm{h}} 53 \mathrm{I}$ & $\mathrm{a}=25.8 \AA ; \mathrm{c}=3.9 \AA$ \\
\hline $\begin{array}{l}\text { [a] Temperature at } \\
\text { [b] DSC data of the } \\
\text { are read at the max } \\
\text { hexagonal columna } \\
\text { [c] } a \text { : lattice consta }\end{array}$ & $\begin{array}{l}\text { ich } 2 \% \text { mass } \\
\text { cond heating } \\
\mathrm{m} \text { of the cor } \\
\text { lesophase. } \\
\text { of the } \mathrm{Col}_{\text {h. }} \text {. }\end{array}$ & $\begin{array}{l}\text { oss is detected in the th } \\
\text { process at a rate of } 10^{\circ} \\
\text { esponding peaks. I: iso } \\
\text { mean stacking distance }\end{array}$ & $\begin{array}{l}\text { mogravimetric curve. } \\
\text { /min. Temperatures }\left({ }^{\circ} \mathrm{C}\right) \\
\text { opic liquid, } \mathrm{Col}_{\mathrm{h}} \text { : }\end{array}$ \\
\hline
\end{tabular}

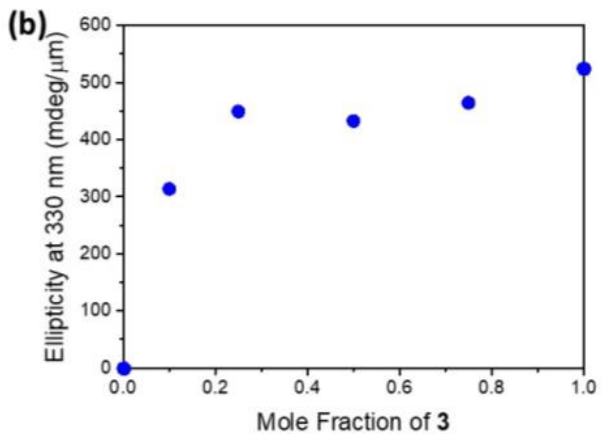

Figure S12. (a) Thermal stability, liquid crystal properties and X-Ray structural parameters of mixtures of (S)-3 and 1. (b) Ellipticity at $330 \mathrm{~nm}$ in mixtures of (S)-3 and $\mathbf{1}$ with different composition. 

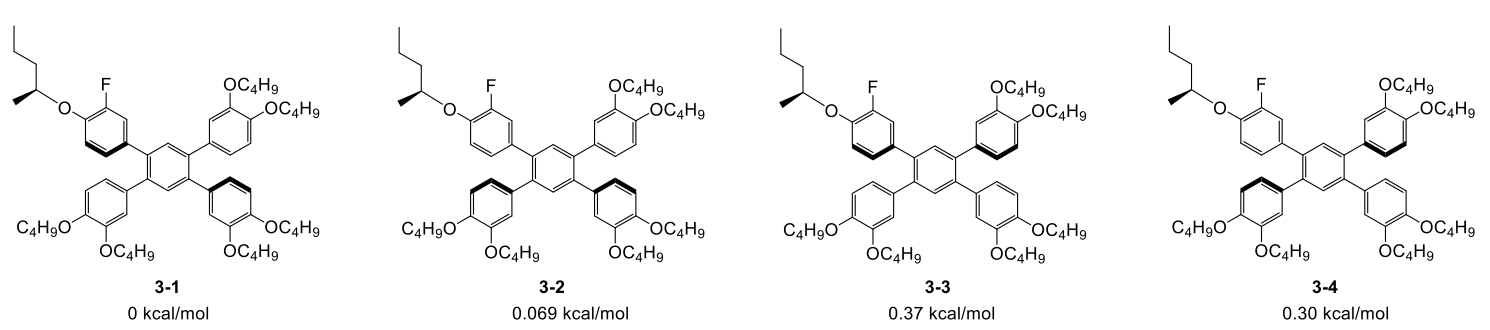

Figure S13. Four representative conformers of $(S)-3$ and their relative energies.

The coordinates of optimized structures

3-1

C

C

$\mathrm{C}$

C

$\mathrm{C}$

C

C

C $\quad-1.61305900 \quad 2.12779100 \quad-0.20549600$

$0.86103800-1.42018100$

$-0.39767300$

$1.43895900-0.12950600-0.38299800$

$0.58494300 \quad 0.97859400-0.32784100$

$\begin{array}{lll}-0.81217600 & 0.87871600 & -0.30921900\end{array}$

C $\quad-1.38972000-0.41166600-0.36607400$

C $\quad-0.53558500-1.52074200-0.39708800$

C $\quad-2.85870900-0.64993100 \quad-0.41548100$

C $\quad 1.66178300 \quad-2.67384400-0.38381100$

C $\quad 2.90642400 \quad 0.10918400 \quad-0.44915200$

C $\quad-3.67271200-0.00679600-1.35393400$

C $\quad-5.03910500-0.28251900-1.40007000$

C $\quad-5.62058300-1.18495000 \quad-0.51437100$

C $\quad-4.81172800 \quad-1.83256800 \quad 0.44526300$

C $\quad-3.44148900-1.56525200 \quad 0.47908800$

C $\quad-1.33015900 \quad 3.22442800 \quad-1.03715100$

C $\quad-2.04923400 \quad 4.41038100 \quad-0.94916500$

C $\quad-3.08961300 \quad 4.53335400 \quad-0.00207200$

C $\quad-3.36583700 \quad 3.45380900 \quad 0.84299300$

$\begin{array}{llll}\text { C } & -2.63663400 & 2.26884900 & 0.73821500\end{array}$

C $\quad 3.70310400 \quad-0.49184800 \quad-1.43543500$

C $\quad 5.06703300 \quad-0.22203700 \quad-1.50554900$

C $\quad 5.67438300 \quad 0.64218400 \quad-0.59329900$

C $\quad 4.89040200 \quad 1.22988500 \quad 0.42076600$

C $\quad 3.52219100 \quad 0.96945000 \quad 0.47174100$

C $\quad 1.37243900 \quad-3.70581400 \quad-1.28819200$

C $\quad 2.10142700 \quad-4.88505000 \quad-1.26273800$

C $\quad 3.12272800 \quad-5.11415500-0.33259500$

C $\quad 3.39936000 \quad-4.08084600 \quad 0.57205200$

C $\quad 2.69287800 \quad-2.88315900 \quad 0.54636100$

O $\quad 3.92318100-6.21773200-0.31008600$

F $\quad \begin{array}{llll}\text { C } & 1.79380700 & -5.84588900 & -2.17164300\end{array}$

C $\quad 3.39921100 \quad-7.57365600 \quad-0.18959200$ 


\begin{tabular}{|c|c|c|c|}
\hline C & 3.59513400 & -8.29393500 & -1.52635800 \\
\hline C & 3.34958600 & -9.80710400 & -1.46125500 \\
\hline C & 3.69203400 & -10.50669600 & -2.78079700 \\
\hline C & 1.97967600 & -7.63659600 & 0.36706200 \\
\hline $\mathrm{O}$ & -6.97955700 & -1.38279900 & -0.54881900 \\
\hline C & -11.03096800 & -4.10857400 & -1.27797200 \\
\hline C & -9.50444900 & -4.05370400 & -1.39439800 \\
\hline C & -8.92343400 & -2.72203700 & -0.90523700 \\
\hline C & -7.40783000 & -2.67359100 & -1.01279700 \\
\hline 0 & -5.45878800 & -2.68396500 & 1.29539500 \\
\hline C & -5.94266300 & -5.87456200 & 5.01600000 \\
\hline C & -4.97347900 & -4.99168200 & 4.22350700 \\
\hline C & -5.66836300 & -4.22923100 & 3.08934800 \\
\hline C & -4.70418600 & -3.35568300 & 2.30069300 \\
\hline O & -3.75807500 & 5.72305900 & 0.00234200 \\
\hline C & -7.21506700 & 8.97382900 & 1.32134400 \\
\hline C & -6.63289900 & 7.57747500 & 1.56216600 \\
\hline C & -5.43044800 & 7.27911400 & 0.65906200 \\
\hline C & -4.85456200 & 5.89139000 & 0.89806500 \\
\hline 0 & -1.77669100 & 5.42288700 & -1.83757500 \\
\hline C & -0.15786600 & 9.92819200 & -3.04036900 \\
\hline C & -0.31568900 & 8.89846200 & -1.91709900 \\
\hline C & -0.99284100 & 7.60767800 & -2.39163600 \\
\hline C & -1.15398100 & 6.58885000 & -1.27492300 \\
\hline O & 5.42592600 & 2.10627600 & 1.33836800 \\
\hline C & 9.21172600 & 3.41891900 & 4.22398700 \\
\hline C & 8.35474600 & 2.25431200 & 3.71829300 \\
\hline C & 7.20867900 & 2.72033300 & 2.81328700 \\
\hline C & 6.41119000 & 1.56042000 & 2.23653100 \\
\hline O & 7.03414000 & 0.83556600 & -0.67049400 \\
\hline C & 10.92886600 & 3.81018800 & -0.08480900 \\
\hline C & 9.49609900 & 3.69878000 & -0.61494000 \\
\hline C & 8.93198900 & 2.27963300 & -0.48245300 \\
\hline C & 7.47856600 & 2.18312900 & -0.92082000 \\
\hline $\mathrm{H}$ & 1.03089200 & 1.96877400 & -0.29753500 \\
\hline $\mathrm{H}$ & -0.98132800 & -2.51140200 & -0.42172400 \\
\hline $\mathrm{H}$ & -3.23728300 & 0.70586700 & -2.04607900 \\
\hline $\mathrm{H}$ & -5.68639500 & 0.20393500 & -2.12343300 \\
\hline $\mathrm{H}$ & -2.80872000 & -2.04321900 & 1.21774000 \\
\hline $\mathrm{H}$ & -0.55551100 & 3.16131600 & -1.79538900 \\
\hline $\mathrm{H}$ & -4.15474500 & 3.52356200 & 1.58291600 \\
\hline $\mathrm{H}$ & -2.87601200 & 1.44097500 & 1.39739000 \\
\hline $\mathrm{H}$ & 3.24855900 & -1.16563900 & -2.15421600 \\
\hline $\mathrm{H}$ & 5.69020100 & -0.67126800 & -2.27273900 \\
\hline $\mathrm{H}$ & 2.94576300 & 1.43707900 & 1.26420000 \\
\hline $\mathrm{H}$ & 0.59905900 & -3.59266600 & -2.04116100 \\
\hline $\mathrm{H}$ & 4.19426400 & -4.24761500 & 1.29222800 \\
\hline $\mathrm{H}$ & 2.93766600 & -2.10011200 & 1.25604400 \\
\hline
\end{tabular}




\begin{tabular}{|c|c|c|c|}
\hline $\mathrm{H}$ & 4.07538500 & -8.02780700 & 0.54705100 \\
\hline $\mathrm{H}$ & 2.94231500 & -7.83766500 & -2.27677300 \\
\hline $\mathrm{H}$ & 4.62751100 & -8.10533000 & -1.84883400 \\
\hline $\mathrm{H}$ & 3.94862100 & -10.24314300 & -0.64826500 \\
\hline $\mathrm{H}$ & 2.29933500 & -10.00518700 & -1.20982400 \\
\hline $\mathrm{H}$ & 3.09155400 & -10.10361300 & -3.60539200 \\
\hline $\mathrm{H}$ & 4.74797000 & -10.36285900 & -3.04005600 \\
\hline $\mathrm{H}$ & 1.74782400 & -8.66852900 & 0.64893800 \\
\hline $\mathrm{H}$ & 1.88511700 & -7.01258200 & 1.26149000 \\
\hline $\mathrm{H}$ & 1.24200300 & -7.31167000 & -0.37102100 \\
\hline $\mathrm{H}$ & -11.35083000 & -3.97738100 & -0.23694900 \\
\hline $\mathrm{H}$ & -11.49926400 & -3.31298000 & -1.87028100 \\
\hline $\mathrm{H}$ & -9.20987600 & -4.21934000 & -2.44043100 \\
\hline $\mathrm{H}$ & -9.06219300 & -4.87864300 & -0.81778100 \\
\hline $\mathrm{H}$ & -9.20196600 & -2.55097700 & 0.14283500 \\
\hline $\mathrm{H}$ & -9.34628200 & -1.88999900 & -1.48360000 \\
\hline $\mathrm{H}$ & -7.08255400 & -2.81055700 & -2.05581500 \\
\hline $\mathrm{H}$ & -6.95072800 & -3.46366500 & -0.40525400 \\
\hline $\mathrm{H}$ & -6.74349000 & -5.27427700 & 5.46452100 \\
\hline $\mathrm{H}$ & -6.41338700 & -6.62299900 & 4.36702400 \\
\hline $\mathrm{H}$ & -4.16690900 & -5.61208600 & 3.80783400 \\
\hline $\mathrm{H}$ & -4.48990700 & -4.27525600 & 4.90252300 \\
\hline $\mathrm{H}$ & -6.46587500 & -3.59285900 & 3.49371400 \\
\hline $\mathrm{H}$ & -6.14875300 & -4.93500000 & 2.39893600 \\
\hline $\mathrm{H}$ & -3.91402300 & -3.96679400 & 1.83835200 \\
\hline $\mathrm{H}$ & -4.21702200 & -2.62049300 & 2.95897400 \\
\hline $\mathrm{H}$ & -6.46590600 & 9.75138100 & 1.51423400 \\
\hline $\mathrm{H}$ & -7.54960500 & 9.08647900 & 0.28299500 \\
\hline $\mathrm{H}$ & -7.41207800 & 6.82062700 & 1.39457500 \\
\hline $\mathrm{H}$ & -6.33323300 & 7.48105900 & 2.61541000 \\
\hline $\mathrm{H}$ & -4.63938100 & 8.02196500 & 0.82599200 \\
\hline $\mathrm{H}$ & -5.72018900 & 7.35976900 & -0.39640900 \\
\hline $\mathrm{H}$ & -5.61341000 & 5.11669100 & 0.71213400 \\
\hline $\mathrm{H}$ & -4.51350300 & 5.78506700 & 1.93922700 \\
\hline $\mathrm{H}$ & 0.44801400 & 9.52491500 & -3.86095400 \\
\hline $\mathrm{H}$ & -1.13311100 & 10.20868600 & -3.45662200 \\
\hline $\mathrm{H}$ & -0.90057800 & 9.33738200 & -1.09621600 \\
\hline $\mathrm{H}$ & 0.67154800 & 8.66054100 & -1.49602900 \\
\hline $\mathrm{H}$ & -0.41111900 & 7.15230900 & -3.20393300 \\
\hline $\mathrm{H}$ & -1.98604400 & 7.83109800 & -2.80259600 \\
\hline $\mathrm{H}$ & -1.77367000 & 6.99379000 & -0.46588400 \\
\hline $\mathrm{H}$ & -0.17608400 & 6.31015900 & -0.85236400 \\
\hline $\mathrm{H}$ & 9.66676700 & 3.96169700 & 3.38572300 \\
\hline $\mathrm{H}$ & 8.60766100 & 4.13587100 & 4.79323400 \\
\hline $\mathrm{H}$ & 7.94740400 & 1.69443200 & 4.57162500 \\
\hline $\mathrm{H}$ & 8.98953600 & 1.54827600 & 3.16385300 \\
\hline $\mathrm{H}$ & 7.61472500 & 3.30801400 & 1.97982000 \\
\hline $\mathrm{H}$ & 6.53340300 & 3.38758900 & 3.36434700 \\
\hline
\end{tabular}




$\begin{array}{cccc}\mathrm{H} & 5.89780100 & 0.99622100 & 3.02920200 \\ \mathrm{H} & 7.07022800 & 0.87101700 & 1.69854200 \\ \mathrm{H} & 10.97348300 & 3.54272200 & 0.97866000 \\ \mathrm{H} & 11.60364500 & 3.13455700 & -0.62431100 \\ \mathrm{H} & 9.46361800 & 4.01336300 & -1.66734900 \\ \mathrm{H} & 8.84924300 & 4.39838300 & -0.06618400 \\ \mathrm{H} & 8.99848600 & 1.95396500 & 0.56373500 \\ \mathrm{H} & 9.53493500 & 1.57245500 & -1.06670500 \\ \mathrm{H} & 7.37015600 & 2.40083500 & -1.99349300 \\ \mathrm{H} & 6.86011700 & 2.89358800 & -0.36305000 \\ \mathrm{H} & -8.07407600 & 9.16651400 & 1.97358100 \\ \mathrm{H} & -11.42636900 & -5.06736500 & -1.63216300 \\ \mathrm{H} & -5.42911300 & -6.40695600 & 5.82427200 \\ \mathrm{H} & 3.50362000 & -11.58484200 & -2.72400800 \\ \mathrm{H} & 11.31796200 & 4.82895100 & -0.19092900 \\ \mathrm{H} & 10.02041900 & 3.06906500 & 4.87526400 \\ \mathrm{H} & 0.32917800 & 10.84199800 & -2.68142300\end{array}$

$\begin{array}{lrrr}3-2 & & & \\ \text { C } & -1.15482000 & -1.23991900 & 0.19083700 \\ \text { C } & -1.41748100 & 0.09741100 & -0.18622900 \\ \text { C } & -0.32958500 & 0.96650500 & -0.33434800 \\ \text { C } & 1.00246100 & 0.58344800 & -0.13133400 \\ \text { C } & 1.26314600 & -0.75443200 & 0.24907600 \\ \text { C } & 0.17597200 & -1.62729700 & 0.38817300 \\ \text { C } & 2.62573100 & -1.26769200 & 0.55817800 \\ \text { C } & 2.06757800 & 1.59367900 & -0.36909600 \\ \text { C } & -2.22994700 & -2.23879300 & 0.43301100 \\ \text { C } & -2.78343100 & 0.61533900 & -0.47140000 \\ \text { C } & 3.48302900 & -0.58950400 & 1.43161600 \\ \text { C } & 4.73908400 & -1.11670700 & 1.72977400 \\ \text { C } & 5.16879300 & -2.31385700 & 1.16511500 \\ \text { C } & 4.31535000 & -3.00651500 & 0.27807100 \\ \text { C } & 3.05454200 & -2.48052300 & -0.01080900 \\ \text { C } & 1.95011600 & 2.88247900 & 0.17804300 \\ \text { C } & 2.91184000 & 3.86030700 & -0.04388800 \\ \text { C } & 4.04007200 & 3.56753100 & -0.84088000 \\ \text { C } & 4.15742700 & 2.29266700 & -1.40443900 \\ \text { C } & 3.18355800 & 1.32167600 & -1.16814400 \\ \text { C } & -3.66731600 & -0.05913000 & -1.32110100 \\ \text { C } & -4.93456900 & 0.46606500 & -1.57414200 \\ \text { C } & -5.34055200 & 1.66927000 & -1.00504600 \\ \text { C } & -4.44890000 & 2.37328000 & -0.16541500 \\ \text { C } & -3.18830100 & 1.83557900 & 0.09928300 \\ \text { C } & -2.17242800 & -3.49686000 & -0.18385900 \\ \text { C } & -3.17308600 & -4.42924200 & 0.04283100 \\ \text { C } & -4.27099400 & -4.16154500 & 0.86833900 \\ \text { C } & -4.31166700 & -2.91006100 & 1.49357200\end{array}$




\begin{tabular}{|c|c|c|c|}
\hline C & -3.31130800 & -1.96584000 & 1.28523500 \\
\hline $\mathrm{O}$ & -5.24454700 & -5.07582100 & 1.14688400 \\
\hline$F$ & -3.09994300 & -5.62473900 & -0.59479600 \\
\hline C & -6.14090900 & -5.57623700 & 0.10736600 \\
\hline C & -5.85957500 & -7.06852400 & -0.08350700 \\
\hline C & -6.90583000 & -7.79844800 & -0.93543200 \\
\hline C & -6.65747300 & -9.30952100 & -0.98989500 \\
\hline C & -6.10331300 & -4.75979300 & -1.18149100 \\
\hline $\mathrm{O}$ & 6.43739000 & -2.76751300 & 1.43145400 \\
\hline C & 9.68200100 & -6.01324000 & 3.24920000 \\
\hline C & 8.20635700 & -5.60951300 & 3.16838000 \\
\hline C & 7.98516000 & -4.35184400 & 2.32031600 \\
\hline C & 6.51967900 & -3.95719600 & 2.23346800 \\
\hline O & 4.81543500 & -4.16279600 & -0.24983000 \\
\hline C & 4.78053800 & -8.35547300 & -2.83270200 \\
\hline C & 3.99434800 & -7.09663600 & -2.45334200 \\
\hline C & 4.77476200 & -6.18261200 & -1.50145400 \\
\hline C & 3.99058000 & -4.93683800 & -1.11717100 \\
\hline $\mathrm{O}$ & 4.94441700 & 4.57792700 & -0.99208400 \\
\hline C & 9.07823400 & 6.77404500 & -2.52059500 \\
\hline C & 8.24952700 & 5.48576200 & -2.53815100 \\
\hline C & 6.94498900 & 5.61690300 & -1.74354800 \\
\hline C & 6.12237800 & 4.33727900 & -1.75913800 \\
\hline O & 2.77630600 & 5.08022100 & 0.57516800 \\
\hline C & 1.98959900 & 9.95277300 & 0.61394600 \\
\hline C & 2.06008000 & 8.68946100 & -0.24979600 \\
\hline C & 2.39652300 & 7.43600600 & 0.56608900 \\
\hline C & 2.47368700 & 6.18398300 & -0.29280200 \\
\hline O & -4.90920100 & 3.55708700 & 0.33623200 \\
\hline C & -4.66405500 & 7.87653500 & 2.69207700 \\
\hline C & -3.92061400 & 6.58641200 & 2.33215700 \\
\hline C & -4.77349200 & 5.63376800 & 1.48623800 \\
\hline C & -4.03189500 & 4.35475600 & 1.12812400 \\
\hline O & -6.57420600 & 2.18463400 & -1.31807500 \\
\hline C & -11.05622100 & 3.87101500 & -0.10555300 \\
\hline C & -9.84464200 & 3.05192700 & 0.35041500 \\
\hline C & -8.74008400 & 2.99056200 & -0.71080800 \\
\hline C & -7.53299900 & 2.19161700 & -0.24729700 \\
\hline $\mathrm{H}$ & -0.52468000 & 1.97727600 & -0.68096200 \\
\hline $\mathrm{H}$ & 0.37060200 & -2.64025100 & 0.72972400 \\
\hline $\mathrm{H}$ & 3.16739300 & 0.34701500 & 1.87836300 \\
\hline $\mathrm{H}$ & 5.41680600 & -0.60505700 & 2.40637700 \\
\hline $\mathrm{H}$ & 2.39520800 & -2.99099100 & -0.70310200 \\
\hline $\mathrm{H}$ & 1.10970900 & 3.13939600 & 0.81584400 \\
\hline $\mathrm{H}$ & 5.01066600 & 2.04357400 & -2.02463400 \\
\hline $\mathrm{H}$ & 3.30044600 & 0.33710200 & -1.60868400 \\
\hline $\mathrm{H}$ & -3.36820100 & -0.99663600 & -1.77729700 \\
\hline $\mathrm{H}$ & -5.63293900 & -0.04292400 & -2.23162300 \\
\hline
\end{tabular}




\begin{tabular}{|c|c|c|c|}
\hline $\mathrm{H}$ & -2.50318500 & 2.35113900 & 0.76191000 \\
\hline $\mathrm{H}$ & -1.36219400 & -3.75432300 & -0.85842600 \\
\hline $\mathrm{H}$ & -5.15253300 & -2.70264900 & 2.14805600 \\
\hline $\mathrm{H}$ & -3.37357100 & -0.99840400 & 1.77214900 \\
\hline $\mathrm{H}$ & -7.13644700 & -5.46397900 & 0.55802400 \\
\hline $\mathrm{H}$ & -4.86341600 & -7.18640800 & -0.52262100 \\
\hline $\mathrm{H}$ & -5.81992600 & -7.52335800 & 0.91490300 \\
\hline $\mathrm{H}$ & -7.90986500 & -7.60558200 & -0.52983500 \\
\hline $\mathrm{H}$ & -6.90463600 & -7.39581700 & -1.95692300 \\
\hline $\mathrm{H}$ & -5.66955900 & -9.52920500 & -1.41286000 \\
\hline $\mathrm{H}$ & -6.69250100 & -9.75081800 & 0.01363300 \\
\hline $\mathrm{H}$ & -6.93990900 & -5.05628300 & -1.82188600 \\
\hline $\mathrm{H}$ & -6.20039000 & -3.68963000 & -0.97307500 \\
\hline $\mathrm{H}$ & -5.17485500 & -4.92784800 & -1.73468100 \\
\hline $\mathrm{H}$ & 10.08868900 & -6.21801500 & 2.25130500 \\
\hline $\mathrm{H}$ & 10.28490100 & -5.21191700 & 3.69364900 \\
\hline $\mathrm{H}$ & 7.81581500 & -5.44134900 & 4.18212100 \\
\hline $\mathrm{H}$ & 7.62071900 & -6.44003400 & 2.74901900 \\
\hline $\mathrm{H}$ & 8.36008200 & -4.51287100 & 1.30112900 \\
\hline $\mathrm{H}$ & 8.55473700 & -3.51028600 & 2.73624100 \\
\hline $\mathrm{H}$ & 6.10687500 & -3.75021800 & 3.23299700 \\
\hline $\mathrm{H}$ & 5.92870300 & -4.76076100 & 1.77858200 \\
\hline $\mathrm{H}$ & 5.72303700 & -8.09575000 & -3.32987300 \\
\hline $\mathrm{H}$ & 5.02745300 & -8.94770800 & -1.94320500 \\
\hline $\mathrm{H}$ & 3.04235300 & -7.38523100 & -1.98570500 \\
\hline $\mathrm{H}$ & 3.73223800 & -6.53869100 & -3.36336100 \\
\hline $\mathrm{H}$ & 5.72025200 & -5.87219400 & -1.96439500 \\
\hline $\mathrm{H}$ & 5.03711600 & -6.72815900 & -0.58547500 \\
\hline $\mathrm{H}$ & 3.05382900 & -5.20782100 & -0.60662400 \\
\hline $\mathrm{H}$ & 3.72793000 & -4.35069200 & -2.01091100 \\
\hline $\mathrm{H}$ & 8.51671900 & 7.60950000 & -2.95615900 \\
\hline $\mathrm{H}$ & 9.34712400 & 7.05446800 & -1.49499100 \\
\hline $\mathrm{H}$ & 8.84664400 & 4.65955200 & -2.12717400 \\
\hline $\mathrm{H}$ & 8.01940900 & 5.21077100 & -3.57723700 \\
\hline $\mathrm{H}$ & 6.33385800 & 6.43159000 & -2.15394400 \\
\hline $\mathrm{H}$ & 7.16154600 & 5.88063300 & -0.70047600 \\
\hline $\mathrm{H}$ & 6.69018000 & 3.50203800 & -1.32270900 \\
\hline $\mathrm{H}$ & 5.85322100 & 4.05925200 & -2.78951800 \\
\hline $\mathrm{H}$ & 1.22190200 & 9.85517800 & 1.39134200 \\
\hline $\mathrm{H}$ & 2.94657500 & 10.13925500 & 1.11640000 \\
\hline $\mathrm{H}$ & 2.81396100 & 8.82503700 & -1.03827100 \\
\hline $\mathrm{H}$ & 1.10060900 & 8.54389000 & -0.76635600 \\
\hline $\mathrm{H}$ & 1.64298900 & 7.28157300 & 1.34982600 \\
\hline $\mathrm{H}$ & 3.35947700 & 7.56524900 & 1.07709100 \\
\hline $\mathrm{H}$ & 3.25134400 & 6.28577400 & -1.05929700 \\
\hline $\mathrm{H}$ & 1.51465000 & 5.99553900 & -0.80042800 \\
\hline $\mathrm{H}$ & -4.96733500 & 8.42032700 & 1.78919400 \\
\hline $\mathrm{H}$ & -5.57141800 & 7.66046900 & 3.26914000 \\
\hline
\end{tabular}




$\begin{array}{cccc}H & -3.60368600 & 6.07567000 & 3.25238500 \\ \mathrm{H} & -2.99916100 & 6.83391200 & 1.78629000 \\ \mathrm{H} & -5.08574400 & 6.12812900 & 0.55749900 \\ \mathrm{H} & -5.69206800 & 5.36854800 & 2.02607400 \\ \mathrm{H} & -3.73709900 & 3.80790800 & 2.03678400 \\ \mathrm{H} & -3.11638400 & 4.58044100 & 0.56082200 \\ \mathrm{H} & -10.76804200 & 4.90391800 & -0.33612500 \\ \mathrm{H} & -11.50263800 & 3.44266800 & -1.01132300 \\ \mathrm{H} & -10.16599000 & 2.03186500 & 0.60471300 \\ \mathrm{H} & -9.43730200 & 3.48367200 & 1.27580300 \\ \mathrm{H} & -8.40363600 & 4.00387000 & -0.96575000 \\ \mathrm{H} & -9.12838500 & 2.54473900 & -1.63609700 \\ \mathrm{H} & -7.81335100 & 1.15431300 & -0.00615900 \\ \mathrm{H} & -7.09427800 & 2.64446800 & 0.64951600 \\ \mathrm{H} & 10.00631000 & 6.65985600 & -3.09158600 \\ \mathrm{H} & 9.81893600 & -6.91354900 & 3.85886900 \\ \mathrm{H} & 4.20728200 & -8.99496100 & -3.51301500 \\ \mathrm{H} & -7.40729000 & -9.81751900 & -1.60699500 \\ \mathrm{H} & -11.83230000 & 3.90357400 & 0.66751900 \\ \mathrm{H} & -4.03662300 & 8.54510900 & 3.29186900 \\ \mathrm{H} & 1.74803200 & 10.83686200 & 0.01309300\end{array}$

3-3

C

$\begin{array}{lll}1.62917300 & 1.14843500 & -0.19971800\end{array}$

C $\quad 1.76355100 \quad-0.20704200 \quad 0.18252400$

C $\quad 0.59798900 \quad-0.96975900 \quad 0.33010800$

C $\quad-0.69222200 \quad-0.46423700 \quad 0.12625300$

$\begin{array}{llll}\text { C } & -0.82556300 & 0.89128900 & -0.25564800\end{array}$

C $\quad 0.33892600 \quad 1.65625600 \quad-0.39981000$

C $\quad-2.13440300 \quad 1.53127400-0.56106800$

C $\quad-1.84760800 \quad-1.37000200 \quad 0.36321400$

C $\quad 2.78694300 \quad 2.04714000 \quad-0.45222000$

C $\quad 3.06924200 \quad-0.85532800 \quad 0.48267200$

C $\quad-3.04952900 \quad 0.94596200 \quad-1.44279200$

C $\quad-4.25720300 \quad 1.58409700 \quad-1.72334200$

C $\quad-4.57320600 \quad 2.81156700 \quad-1.14826600$

C $\quad-3.65003900 \quad 3.42308600 \quad-0.27181100$

C $\quad-2.44716900 \quad 2.77450800 \quad 0.01679800$

C $\quad-1.85434800-2.66086600 \quad-0.19158000$

C $\quad-2.89913100 \quad-3.54788200 \quad 0.03602600$

C $\quad-3.98800900-3.15760900 \quad 0.84607700$

C $\quad-3.99314600 \quad-1.87310200 \quad 1.39931600$

C $\quad-2.93575100 \quad-0.99455200 \quad 1.15892200$

C $\quad 4.00748000 \quad-0.26724000 \quad 1.33784300$

C $\quad 5.19805600-0.93107000 \quad 1.63365000$

C $\quad 5.48158200-2.17833700 \quad 1.08540500$

C $\quad 4.54692500-2.77986400 \quad 0.21364300$

C $\quad 3.35312700 \quad-2.11578000 \quad-0.07392700$ 


\begin{tabular}{|c|c|c|c|}
\hline C & 2.80547400 & 3.33914000 & 0.09294300 \\
\hline C & 3.86958000 & 4.19151000 & -0.15891700 \\
\hline C & 4.94537300 & 3.82386600 & -0.97608700 \\
\hline C & 4.91464900 & 2.53440500 & -1.52308000 \\
\hline C & 3.86482600 & 1.65987800 & -1.26475000 \\
\hline O & 6.05306900 & 4.58821500 & -1.19330100 \\
\hline $\mathrm{F}$ & 3.84671900 & 5.42712800 & 0.40509300 \\
\hline C & 5.98862900 & 5.93195200 & -1.75742900 \\
\hline C & 6.35792900 & 6.93683400 & -0.66272300 \\
\hline C & 6.61243300 & 8.35785300 & -1.18185800 \\
\hline C & 7.11826800 & 9.29531000 & -0.08038000 \\
\hline C & 4.68029000 & 6.23270700 & -2.48287600 \\
\hline O & -5.75141500 & 3.42854800 & -1.49149500 \\
\hline C & -10.32412100 & 5.05211400 & -0.58243400 \\
\hline C & -9.08719400 & 4.36085700 & 0.00001700 \\
\hline C & -7.95923900 & 4.20824000 & -1.02704700 \\
\hline C & -6.73455100 & 3.51832400 & -0.44771400 \\
\hline O & -4.02053100 & 4.63476100 & 0.23828200 \\
\hline C & -3.51729600 & 8.82872500 & 2.77384700 \\
\hline C & -2.86405000 & 7.49987900 & 2.38116000 \\
\hline C & -3.76049800 & 6.65056400 & 1.47256600 \\
\hline C & -3.11171400 & 5.32891500 & 1.08945900 \\
\hline 0 & -4.96416100 & -4.09394700 & 1.02661600 \\
\hline C & -9.23516500 & -5.95037900 & 2.62159400 \\
\hline C & -8.30747500 & -4.73138600 & 2.62519500 \\
\hline C & -7.03171000 & -4.96434800 & 1.80713900 \\
\hline C & -6.10881400 & -3.75493000 & 1.80621500 \\
\hline O & -2.82712600 & -4.81346000 & -0.49522200 \\
\hline C & -4.32120700 & -8.43614500 & -3.49745000 \\
\hline C & -4.50115300 & -6.96790200 & -3.09889800 \\
\hline C & -3.56859500 & -6.54881500 & -1.95673900 \\
\hline C & -3.75454900 & -5.09422000 & -1.55606200 \\
\hline O & 4.90297500 & -3.99399200 & -0.29989900 \\
\hline C & 4.32713900 & -8.19169000 & -2.81432100 \\
\hline C & 3.70461300 & -6.84228600 & -2.44242200 \\
\hline C & 4.60535400 & -6.01477400 & -1.51802400 \\
\hline C & 3.98172400 & -4.67785200 & -1.14648600 \\
\hline $\mathrm{O}$ & 6.68824700 & -2.77506500 & 1.35614300 \\
\hline C & 9.52527300 & -6.32094000 & 3.29490400 \\
\hline C & 8.10541800 & -5.75763300 & 3.17835600 \\
\hline C & 8.03915300 & -4.50308300 & 2.29979600 \\
\hline C & 6.62802700 & -3.95040800 & 2.18109100 \\
\hline $\mathrm{H}$ & 0.69671100 & -1.99339000 & 0.67967200 \\
\hline $\mathrm{H}$ & 0.23859500 & 2.68004600 & -0.74929600 \\
\hline $\mathrm{H}$ & -2.82076300 & -0.01073000 & -1.89981400 \\
\hline $\mathrm{H}$ & -4.97916800 & 1.14655500 & -2.40619300 \\
\hline $\mathrm{H}$ & -1.73713600 & 3.22072800 & 0.70325800 \\
\hline $\mathrm{H}$ & -1.03932700 & -2.99534900 & -0.82625200 \\
\hline
\end{tabular}




\begin{tabular}{|c|c|c|c|}
\hline $\mathrm{H}$ & -4.81421000 & -1.55106700 & 2.02938500 \\
\hline $\mathrm{H}$ & -2.96011500 & -0.00343100 & 1.59967200 \\
\hline $\mathrm{H}$ & 3.80421100 & 0.70365900 & 1.77653000 \\
\hline $\mathrm{H}$ & 5.93617100 & -0.49155300 & 2.29759400 \\
\hline $\mathrm{H}$ & 2.63348700 & -2.55701700 & -0.75343000 \\
\hline $\mathrm{H}$ & 2.00805100 & 3.68573100 & 0.74240900 \\
\hline $\mathrm{H}$ & 5.74768700 & 2.23892100 & -2.15309900 \\
\hline r & 3.87482000 & 0.66618000 & -1.69938300 \\
\hline $\mathrm{H}$ & 6.79729900 & 5.92091100 & -2.50036200 \\
\hline $\mathrm{H}$ & 5.56702400 & 6.94608100 & 0.09374400 \\
\hline $\mathrm{H}$ & 7.26361500 & 6.56113900 & -0.16886500 \\
\hline $\mathrm{H}$ & 7.34618300 & 8.32475600 & -2.00066000 \\
\hline $\mathrm{H}$ & 5.69028900 & 8.77038000 & -1.61196600 \\
\hline $\mathrm{H}$ & 6.39472000 & 9.36134200 & 0.74136500 \\
\hline $\mathrm{H}$ & 8.06393500 & 8.93296000 & 0.34075600 \\
\hline $\mathrm{H}$ & 4.79611200 & 7.14732200 & -3.07284100 \\
\hline $\mathrm{H}$ & 4.41949800 & 5.42013200 & -3.16835800 \\
\hline $\mathrm{H}$ & 3.85559600 & 6.38240300 & -1.78141500 \\
\hline $\mathrm{H}$ & -10.07756200 & 6.05640600 & -0.94805300 \\
\hline $\mathrm{H}$ & -10.73041600 & 4.48326800 & -1.42762400 \\
\hline $\mathrm{H}$ & -9.36806100 & 3.37031600 & 0.38559100 \\
\hline $\mathrm{H}$ & -8.71922300 & 4.93231000 & 0.86398800 \\
\hline $\mathrm{H}$ & -7.65672900 & 5.19389200 & -1.40409000 \\
\hline $\mathrm{H}$ & -8.31201700 & 3.63427200 & -1.89400900 \\
\hline $\mathrm{H}$ & -6.98484100 & 2.50515000 & -0.09618200 \\
\hline $\mathrm{H}$ & -6.33289400 & 4.08499200 & 0.40056700 \\
\hline $\mathrm{H}$ & -3.74663200 & 9.43143100 & 1.88674300 \\
\hline $\mathrm{H}$ & -4.45770200 & 8.66101400 & 3.31281600 \\
\hline $\mathrm{H}$ & -2.61638600 & 6.93001100 & 3.28792700 \\
\hline $\mathrm{H}$ & -1.90973000 & 7.69619700 & 1.87243900 \\
\hline $\mathrm{H}$ & -4.00044000 & 7.20178900 & 0.55442300 \\
\hline $\mathrm{H}$ & -4.71518500 & 6.44143500 & 1.97300700 \\
\hline $\mathrm{H}$ & -2.89629700 & 4.72754300 & 1.98592000 \\
\hline $\mathrm{H}$ & -2.16036100 & 5.49929000 & 0.56328300 \\
\hline $\mathrm{H}$ & -9.54595200 & -6.20489000 & 1.60100800 \\
\hline $\mathrm{H}$ & -8.73163900 & -6.82872200 & 3.04327000 \\
\hline $\mathrm{H}$ & -8.03748500 & -4.47795900 & 3.66010300 \\
\hline $\mathrm{H}$ & -8.84461400 & -3.85944000 & 2.22578600 \\
\hline $\mathrm{H}$ & -7.28994300 & -5.20906200 & 0.76842200 \\
\hline $\mathrm{H}$ & -6.47900700 & -5.82506100 & 2.20488500 \\
\hline $\mathrm{H}$ & -5.80398900 & -3.49666500 & 2.83159200 \\
\hline $\mathrm{H}$ & -6.61399600 & -2.87748100 & 1.37452500 \\
\hline $\mathrm{H}$ & -3.29414200 & -8.63023600 & -3.83008500 \\
\hline $\mathrm{H}$ & -4.52520600 & -9.10136800 & -2.64953800 \\
\hline $\mathrm{H}$ & -5.54475400 & -6.79375400 & -2.80047800 \\
\hline $\mathrm{H}$ & -4.32185800 & -6.32577500 & -3.97296600 \\
\hline $\mathrm{H}$ & -2.52118500 & -6.70498800 & -2.24709700 \\
\hline $\mathrm{H}$ & -3.74738200 & -7.17603600 & -1.07365100 \\
\hline
\end{tabular}




$\begin{array}{cccc}H & -4.78149000 & -4.91380200 & -1.21671700 \\ H & -3.55008800 & -4.42254800 & -2.40440800 \\ H & 5.28541000 & -8.05435000 & -3.32978300 \\ H & 4.51576400 & -8.79767700 & -1.91980800 \\ H & 2.73334700 & -7.00762200 & -1.95512000 \\ H & 3.49520300 & -6.26976100 & -3.35706800 \\ H & 5.57394400 & -5.82835900 & -1.99932500 \\ H & 4.81408500 & -6.57324500 & -0.59606000 \\ H & 3.02509500 & -4.82610600 & -0.62288400 \\ H & 3.78179100 & -4.07835800 & -2.04750700 \\ H & 9.92223700 & -6.59323000 & 2.30931300 \\ H & 10.20685500 & -5.58150900 & 3.73284500 \\ H & 7.71977800 & -5.52336600 & 4.18079400 \\ H & 7.43773700 & -6.52765200 & 2.76617500 \\ H & 8.40915900 & -4.72836400 & 1.29107000 \\ H & 8.69270400 & -3.72072400 & 2.70771000 \\ H & 6.22698100 & -3.67892600 & 3.16992000 \\ H & 5.95744500 & -4.69245100 & 1.73188500 \\ \mathrm{H} & -10.13967400 & -5.76632600 & 3.21180900 \\ \mathrm{H} & -11.11780000 & 5.15319600 & 0.16653800 \\ \mathrm{H} & -2.86069800 & 9.42062500 & 3.42108000 \\ \mathrm{H} & 7.28654700 & 10.30886100 & -0.46187100 \\ \mathrm{H} & 9.55129800 & -7.21644100 & 3.92619800 \\ \mathrm{H} & 3.66940700 & -8.76629900 & -3.47590400 \\ \mathrm{H} & -4.99657600 & -8.71612200 & -4.31381800\end{array}$

$\begin{array}{lrrr}3-4 & & & \\ \text { C } & 1.52578800 & 0.98401100 & -0.35090100 \\ \text { C } & 1.54857700 & -0.42973900 & -0.33025000 \\ \text { C } & 0.32568400 & -1.11112600 & -0.31149900 \\ \text { C } & -0.91846700 & -0.46780200 & -0.33515600 \\ \text { C } & -0.93929900 & 0.94506500 & -0.40660000 \\ \text { C } & 0.28360200 & 1.62782700 & -0.40104500 \\ \text { C } & -2.19384200 & 1.74041100 & -0.51004700 \\ \text { C } & -2.14770500 & -1.30216700 & -0.25958300 \\ \text { C } & 2.75772000 & 1.81561200 & -0.29126400 \\ \text { C } & 2.80690500 & -1.22510400 & -0.35256100 \\ \text { C } & -3.16642900 & 1.45017000 & -1.47291400 \\ \text { C } & -4.31926300 & 2.23030000 & -1.56051500 \\ \text { C } & -4.52261500 & 3.31040800 & -0.70694700 \\ \text { C } & -3.54178200 & 3.62342400 & 0.25989500 \\ \text { C } & -2.39381000 & 2.83371100 & 0.35165500 \\ \text { C } & -2.29414400 & -2.42480700 & -1.09177900 \\ \text { C } & -3.41502000 & -3.24289200 & -1.02070800 \\ \text { C } & -4.43828800 & -2.95449100 & -0.09109700 \\ \text { C } & -4.30369200 & -1.83706600 & 0.73881900 \\ \text { C } & -3.17242600 & -1.02420100 & 0.65190900 \\ \text { C } & 3.80023500 & -0.99379500 & -1.31010500\end{array}$




\begin{tabular}{|c|c|c|c|}
\hline C & 4.95789000 & -1.77164000 & -1.31990300 \\
\hline C & 5.14555300 & -2.79219600 & -0.39264200 \\
\hline C & 4.14338900 & -3.04664000 & 0.56977700 \\
\hline C & 2.99060200 & -2.25864300 & 0.58311000 \\
\hline C & 2.94851100 & 2.86395200 & -1.20311600 \\
\hline C & 4.08676500 & 3.65234600 & -1.13087900 \\
\hline C & 5.08843800 & 3.43487500 & -0.17741900 \\
\hline C & 4.88462600 & 2.39318200 & 0.73538900 \\
\hline C & 3.74274100 & 1.60039900 & 0.68578700 \\
\hline $\mathrm{O}$ & 6.19260100 & 4.22346200 & -0.03750700 \\
\hline $\mathrm{F}$ & 4.24304000 & 4.64354300 & -2.04481800 \\
\hline C & 7.18619900 & 4.35755400 & -1.09937700 \\
\hline C & 7.13383300 & 5.79439200 & -1.62459300 \\
\hline C & 8.30698200 & 6.16816100 & -2.53959100 \\
\hline C & 8.28984800 & 7.65011500 & -2.92883000 \\
\hline C & 7.07805000 & 3.28619300 & -2.18074700 \\
\hline O & -5.64136800 & 4.09216600 & -0.86094100 \\
\hline C & -9.84645600 & 6.13458000 & 0.72134500 \\
\hline C & -8.78766800 & 5.05822800 & 0.98057900 \\
\hline C & -7.67232200 & 5.06394600 & -0.07129000 \\
\hline C & -6.61363100 & 4.00573900 & 0.19304700 \\
\hline O & -3.80690700 & 4.70464900 & 1.05114700 \\
\hline C & -2.95428000 & 8.09617100 & 4.52207200 \\
\hline C & -2.41710800 & 6.85294000 & 3.80606600 \\
\hline C & -3.38002500 & 6.33027700 & 2.73394800 \\
\hline C & -2.84262300 & 5.09569200 & 2.02569300 \\
\hline O & -5.49566700 & -3.81713000 & -0.08119800 \\
\hline C & -9.83122400 & -5.67263200 & 1.32744300 \\
\hline C & -8.79811100 & -4.56426100 & 1.55288200 \\
\hline C & -7.59416700 & -4.68561200 & 0.61147900 \\
\hline C & -6.56643600 & -3.58548100 & 0.83072100 \\
\hline O & -3.48483300 & -4.35338400 & -1.82676400 \\
\hline C & -5.43939800 & -7.03301800 & -5.48447300 \\
\hline C & -5.44009200 & -5.67949500 & -4.76673000 \\
\hline C & -4.44760100 & -5.63123100 & -3.59948000 \\
\hline C & -4.45737500 & -4.29067200 & -2.88272000 \\
\hline $\mathrm{O}$ & 4.39297400 & -4.07259300 & 1.43541300 \\
\hline C & 3.46390800 & -7.24481800 & 5.08839000 \\
\hline C & 2.94163200 & -6.04944100 & 4.28520200 \\
\hline C & 3.92789900 & -5.59223400 & 3.20440300 \\
\hline C & 3.40580400 & -4.40511800 & 2.40905000 \\
\hline O & 6.27226400 & -3.57352000 & -0.46857800 \\
\hline C & 10.52855300 & -5.37302400 & 1.26496700 \\
\hline C & 9.41856100 & -4.33655600 & 1.46598500 \\
\hline C & 8.32576600 & -4.42834700 & 0.39461600 \\
\hline C & 7.21925800 & -3.40638900 & 0.59906300 \\
\hline $\mathrm{H}$ & 0.34314200 & -2.19691300 & -0.27740900 \\
\hline $\mathrm{H}$ & 0.26687800 & 2.71373800 & -0.43735700 \\
\hline
\end{tabular}




\begin{tabular}{|c|c|c|c|}
\hline $\mathrm{H}$ & -3.02487300 & 0.61064200 & -2.14504000 \\
\hline $\mathrm{H}$ & -5.08273100 & 2.02342800 & -2.30435800 \\
\hline $\mathrm{H}$ & -1.64075100 & 3.04989500 & 1.10020900 \\
\hline $\mathrm{H}$ & -1.53316700 & -2.67618400 & -1.82433000 \\
\hline $\mathrm{H}$ & -5.07292600 & -1.59613400 & 1.46365200 \\
\hline $\mathrm{H}$ & -3.08901900 & -0.16112000 & 1.30417400 \\
\hline & 3.67024700 & -0.20328200 & -2.04154800 \\
\hline & 5.73746100 & -1.61028600 & -2.05834300 \\
\hline & 2.21995500 & -2.42956000 & 1.32555100 \\
\hline $\mathrm{H}$ & 2.22365900 & 3.06866600 & -1.98441300 \\
\hline $\mathrm{H}$ & 5.64999300 & 2.22858700 & 1.48745800 \\
\hline $\mathrm{H}$ & 3.61626200 & 0.79423300 & 1.40055800 \\
\hline $\mathrm{H}$ & 8.13731100 & 4.21643500 & -0.56833000 \\
\hline $\mathrm{H}$ & 6.18335900 & 5.94574000 & -2.14646000 \\
\hline r & 7.12435500 & 6.46030500 & -0.75170800 \\
\hline $\mathrm{H}$ & 9.25614300 & 5.93145400 & -2.03670900 \\
\hline $\mathrm{H}$ & 8.28095700 & 5.55574300 & -3.45051800 \\
\hline $\mathrm{H}$ & 7.35887900 & 7.90823400 & -3.44817500 \\
\hline $\mathrm{H}$ & 8.36186400 & 8.29119600 & -2.04177600 \\
\hline $\mathrm{H}$ & 7.97114300 & 3.31828900 & -2.81268100 \\
\hline $\mathrm{H}$ & 7.01322400 & 2.28720800 & -1.73809200 \\
\hline $\mathrm{H}$ & 6.20414300 & 3.44785100 & -2.81739400 \\
\hline $\mathrm{H}$ & -9.39844100 & 7.13568100 & 0.72802300 \\
\hline $\mathrm{H}$ & -10.32102700 & 5.99160500 & -0.25712500 \\
\hline $\mathrm{H}$ & -9.26713600 & 4.06909800 & 1.00149400 \\
\hline $\mathrm{H}$ & -8.34961100 & 5.20693700 & 1.97793100 \\
\hline $\mathrm{H}$ & -7.17968400 & 6.04465700 & -0.09435600 \\
\hline $\mathrm{H}$ & -8.09502000 & 4.90017700 & -1.07137300 \\
\hline $\mathrm{H}$ & -7.05563400 & 2.99696900 & 0.19728500 \\
\hline $\mathrm{H}$ & -6.13438400 & 4.17353400 & 1.16470600 \\
\hline $\mathrm{H}$ & -3.12424600 & 8.91556000 & 3.81317300 \\
\hline $\mathrm{H}$ & -3.90939300 & 7.88326700 & 5.01760300 \\
\hline $\mathrm{H}$ & -2.22348500 & 6.05992400 & 4.54229400 \\
\hline $\mathrm{H}$ & -1.44725800 & 7.08548900 & 3.34427500 \\
\hline $\mathrm{H}$ & -3.57155300 & 7.10846000 & 1.98413700 \\
\hline $\mathrm{H}$ & -4.34977700 & 6.08278300 & 3.18558600 \\
\hline $\mathrm{H}$ & -2.67538300 & 4.27775200 & 2.74323900 \\
\hline $\mathrm{H}$ & -1.88147000 & 5.31418200 & 1.53659000 \\
\hline $\mathrm{H}$ & -10.21720000 & -5.64788000 & 0.30114100 \\
\hline $\mathrm{H}$ & -9.38783900 & -6.66233100 & 1.49082300 \\
\hline $\mathrm{H}$ & -8.45146600 & -4.59172500 & 2.59563000 \\
\hline $\mathrm{H}$ & -9.27499000 & -3.58358300 & 1.41397500 \\
\hline $\mathrm{H}$ & -7.92799800 & -4.65028800 & -0.43388800 \\
\hline $\mathrm{H}$ & -7.10088200 & -5.65605800 & 0.75098900 \\
\hline $\mathrm{H}$ & -6.19054100 & -3.60303800 & 1.86486500 \\
\hline $\mathrm{H}$ & -7.01030800 & -2.59441600 & 0.65104100 \\
\hline $\mathrm{H}$ & -4.44714100 & -7.26089300 & -5.89254800 \\
\hline $\mathrm{H}$ & -5.70732600 & -7.84329900 & -4.79537400 \\
\hline
\end{tabular}




$\begin{array}{lrrr}\mathrm{H} & -6.45163000 & -5.46138300 & -4.39546200 \\ \mathrm{H} & -5.19960800 & -4.88307200 & -5.48520300 \\ \mathrm{H} & -3.43009400 & -5.83293600 & -3.95926900 \\ \mathrm{H} & -4.68497600 & -6.41559700 & -2.86901900 \\ \mathrm{H} & -5.45064600 & -4.08028500 & -2.46825400 \\ \mathrm{H} & -4.19223200 & -3.47370400 & -3.57175200 \\ \mathrm{H} & 3.64907000 & -8.10652200 & 4.43569700 \\ \mathrm{H} & 4.40828100 & -6.99993500 & 5.58964400 \\ \mathrm{H} & 2.73125300 & -5.21269800 & 4.96635400 \\ \mathrm{H} & 1.98247800 & -6.31218800 & 3.81726400 \\ \mathrm{H} & 4.13654300 & -6.41499700 & 2.50875500 \\ \mathrm{H} & 4.88710400 & -5.31524800 & 3.66133500 \\ \mathrm{H} & 3.22111300 & -3.54449900 & 3.07009300 \\ \mathrm{H} & 2.45685200 & -4.65579400 & 1.91175800 \\ \mathrm{H} & 10.12527700 & -6.39247400 & 1.29895800 \\ \mathrm{H} & 11.01614600 & -5.24292700 & 0.29111000 \\ \mathrm{H} & 9.85354100 & -3.32695600 & 1.46008500 \\ \mathrm{H} & 8.96772900 & -4.46947100 & 2.45984300 \\ \mathrm{H} & 7.87581700 & -5.42964100 & 0.39894300 \\ \mathrm{H} & 8.76129600 & -4.28321700 & -0.60285400 \\ \mathrm{H} & 7.61934900 & -2.38075500 & 0.57176700 \\ \mathrm{H} & 6.72720300 & -3.55621600 & 1.56736000 \\ \mathrm{H} & -10.68297100 & -5.56959600 & 2.00882400 \\ \mathrm{H} & -10.63436500 & 6.11416500 & 1.48278700 \\ \mathrm{H} & -2.25284400 & 8.45248500 & 5.28461000 \\ \mathrm{H} & 9.12508300 & 7.90017300 & -3.59288400 \\ \mathrm{H} & 11.29927500 & -5.29095400 & 2.03975600 \\ \mathrm{H} & 2.74658600 & -7.55406600 & 5.85667700 \\ \mathrm{H} & -6.15492600 & -7.04773900 & -6.31442900\end{array}$

